\title{
Basic Violet Decolourization Using Alginate Immobilized Nanozirconium Tungestovanadate Matrix as Cation Exchanger
}

\author{
M. F. Elkady, ${ }^{1,2}$ H. Shokry Hassan, ${ }^{3}$ and Eman M. El-Sayed ${ }^{1}$ \\ ${ }^{1}$ Fabrication Technology Research Department, Advanced Technology and New Materials Research Institute (ATNMRI), \\ City of Scientific Research and Technological Applications, Alexandria 21934, Egypt \\ ${ }^{2}$ Chemical and Petrochemical Engineering Department, Egypt-Japan University of Science and Technology, \\ New Borg El-Arab, Alexandria 21934, Egypt \\ ${ }^{3}$ Electronic Materials Researches Department, Advanced Technology and New Materials Researches Institute, \\ City of Scientific Research and Technological Applications, New Borg El-Arab, Alexandria 21934, Egypt
}

Correspondence should be addressed to M. F. Elkady; marwa.f.elkady@gmail.com

Received 9 March 2015; Revised 28 May 2015; Accepted 7 July 2015

Academic Editor: Mu Naushad

Copyright (C) 2015 M. F. Elkady et al. This is an open access article distributed under the Creative Commons Attribution License, which permits unrestricted use, distribution, and reproduction in any medium, provided the original work is properly cited.

\begin{abstract}
As an innovative cation exchange material, nanozirconium tungestovanadate prepared using homogeneous precipitation technique was immobilized into alginate matrix and evaluated for cationic dye decolourization. Physicochemical properties of the prepared composite material were examined to determine its crystallinity, morphology, and ion exchange capacity. The SEM and TEM images of the prepared zirconium tungestovanadate identified that it was prepared in homogeneous structure with spherical shape in average diameter $22 \mathrm{~nm}$. However, the optical microscope image of the composite matrix confirms the good distribution of nanozirconium tungestovanadate into the polymeric matrix. Batch technique was utilized to test the cation exchange efficiency of the prepared composite material toward C.I. basic violet 16 dye decolourization. The optimum immobilized dosage from nanozirconium tungestovanadate into the polymeric matrix to achieve $99 \%$ dye decolourization from $50 \mathrm{ppm}$ initial dye concentration was recorded as $8 \mathrm{~g} / \mathrm{L}$. The dye sorption process onto the prepared material is described as an endothermic process. The dye sorption process at equilibrium was following both Langmuir and Freundlich isotherm models. The kinetic sorption behaviour of dye onto the composite matrix is correlated to pseudo-second-order equation model.
\end{abstract}

\section{Introduction}

Dyes have long been used in dyeing, paper and pulp, textiles, plastics, leather, cosmetics, and food industries. Color stuff discharged from these industries poses certain hazards and environmental problems. These colored compounds are not only aesthetically displeasing but also inhibiting sunlight penetration into the stream and affecting aquatic ecosystem. Dyes usually have complex aromatic molecular structures which make them more stable, resistant to fading, and difficult to biodegrade. Furthermore, many dyes are toxic to some microorganisms and may cause direct destruction or inhibition of their catalytic capabilities [1].

Thus, it would be highly desirable to remove synthetic dyes from industrial effluents prior to their discharge. Numerous efforts have been made to overcome this problem.
Up to now, the main techniques used for the removal of dyes from wastewater are chemical oxidation [2], electrochemical treatment [3], membrane separation [4], photocatalytic degradation [5], microbiological decomposition [6], and so forth. However, these methods have some certain limitations such as high capital investment and operation costs. Besides, many synthetic dyes are chemically stable and resistant to photodegradation and biodegradation [7]. Thus, sorption may be an alternative potential technique for removing dyes from wastewater on account of its relatively high capacity, easy regeneration and flexibility in design and operation, and so forth. Sorption is a process by which atoms, molecules, or ions are retained on the surfaces of solids by chemical or physical bonding.

Sorption techniques have proven successful on lowering dye concentration from industrial effluents using adsorbents 
such as activated carbon, peat, chitin, clay, and others $[8,9]$. Synthetic inorganic ion exchangers become much valuable and effective than these conventional adsorbent materials because of their characteristics [10], since they have excellent stabilities towards thermal and radiation doses. Moreover, they often exhibit specificity towards certain ions [11].

Generally, there are three different techniques used for preparing materials which are sol-gel precipitation, precipitation, and hydrothermal techniques. The precipitation from a homogeneous solution is a technique in which a precipitating agent is generated in the reaction solution by slow chemical reaction. Homogeneously formed precipitates are usually better suited for analysis than the solid formed by the direct addition of a precipitating reagent [10]. Although the nanopowder materials have been verified to be efficient sorbent materials for wastewater treatment processes, the handling of nanopowder materials in the water treatment processes has more difficulties either at the batch or continuous processes. The separation of nanopowder materials after the treatment process represents the main problem of the sorption process using batch technique. Moreover, the decline in the pressure drop and clogging the treatment column during the continuous treatment process that contains the nanopowder as sorbent materials are the main disadvantages of the continuous process treatment using nanomaterials. Accordingly, in order to overcome the main drawbacks of nanomaterials utilization at the water treatment processes, some researchers suggested to immobilized the nanopowder materials into polymeric matrices to facilitate their handling into the treatment processes [11].

In this regard, a novel zirconium tungestovanadate with homogeneous nanosize will be prepared using homogeneous precipitation technique. This nanopowdered material will be immobilized onto alginate polymeric matrix to fabricate composite sheet. The cation exchange performance of the fabricated composite sheet material will be examined toward the decolonization process of C.I. basic violet 16 . The equilibrium and kinetics of the dye sorption process onto nanozirconium tungestovanadate-alginate matrix under different experimental conditions will be modeled.

\section{Experimental}

2.1. Reagents and Solutions. C.I. basic violet 16 was obtained from Ciba Ltd. and used without any purification. The chemical structure of this dye is given in Figure 1. Chemicals utilized for cation exchange composite matrix are supplied from Fluka AG (Buchs, Switzerland) for fine chemicals. All solutions were prepared with distilled water under ambient conditions.

2.2. Nanozirconium Tungestovanadate Preparation Using Homogeneous Precipitation Technique. As an attempt to prepare zirconium tungestovanadate with homogeneous and uniform nanosize structure, the material precipitation takes place in presence of urea as precipitating agents. A uniform spherical nanozirconium tungestovanadate was synthetized through the addition of two separate solutions: one is composed of $50 \mathrm{~mL}$ ammonium metavanadate $(1 \mathrm{M})$ and the other contains $50 \mathrm{~mL}$ sodium tungstate $(0.5 \mathrm{M})$. These

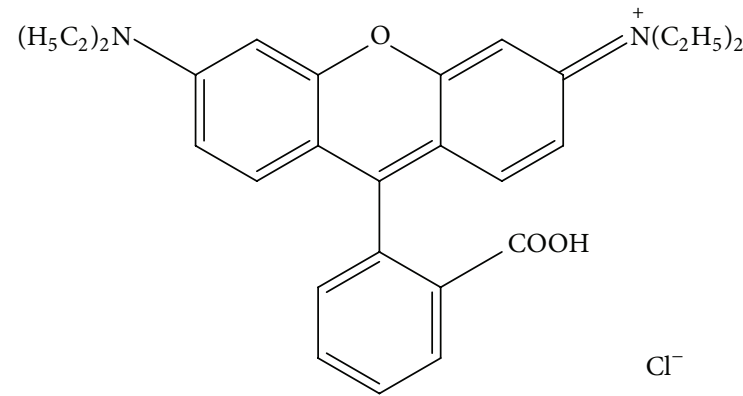

FIgure 1: Chemical structure of C.I. basic violet 16 dyes.

solutions added dropwise to the solution composed of $150 \mathrm{~mL}$ (1 M) zirconium oxychloride contain $2 \mathrm{~g}$ urea. These solutions were mixed with good mixing under heating at $80^{\circ} \mathrm{C}$. After completeness of the addition of the two solutions, the reaction is maintained under heating for about another 30 minutes. The resulting precipitate was filtered and washed several times with hot distilled water. Then the powdered material was dried overnight and the yielded dried powder was ground using manual mortar.

\subsection{Alginate Immobilized Nanozirconium Tungestovanadate} Composite Sheet Matrix. Different weights from the prepared zirconium tungestovanadate was immobilized and welldistributed into $100 \mathrm{~mL}$ sodium alginate solution (2\%wt) using ultra-sonic homogenizer to fabricate different composite matrices with different immobilized weights. The resulting homogeneous slurry that is composed of zirconium tungestovanadate nanopowder that is mixed with alginate solution was poured into dishes and dried under vacuum at $40^{\circ} \mathrm{C}$ overnight. The dried zirconium tungestovanadatealginate sheet matrix was separated from the dishes and cross-linked using the evaporated calcium chloride solution to solidify the composite matrix.

2.4. Characteristics of Synthesized Nanozirconium Tungestovanadate and Alginate Composite Matrix. The cation exchange capacities of the prepared nanozirconium tungestovanadate, pure alginate, and alginate-zirconium tungestovanadate composite matrices were compared using the acid-base titration technique [10]. $0.2 \mathrm{~g}$ from the powder material or sheet was shacked with $50 \mathrm{~mL}$ solution of $1 \mathrm{M}$ sodium chloride for 1 day at room temperature. The released hydrogen ions at the solution were titrated against $0.1 \mathrm{M}$ solution of $\mathrm{NaOH}$ in presence of phenolphthalein indicator:

$$
\mathrm{IEC}=\frac{(V * C)_{\mathrm{NaOH}}}{w},
$$

where $C$ and $V$ are the concentration and volume of $\mathrm{NaOH}$, respectively, and $w$ is the weight of ion exchange material. The crystalline and morphological structures of the prepared nanozirconium tungestovanadate were examined using $\mathrm{X}$ ray diffraction (Shimadzu-7000 diffractometer) and scanning (JEOL JSM 6360 LA, Japan) and transmission electron microscopes (JEOL JEM-1230, Japan), respectively. The fabricated nanozirconium tungestovanadate-alginate composite 
matrix was packed into aluminum holder and subjected to $\mathrm{Cu} \mathrm{Ka}$ radiation beam over $2 \theta$ between $10^{\circ}$ and $80^{\circ}$ to determine its crystalline structure. The morphological structure of the prepared nanozirconium tungestovanadate was determined through either imaging the powder material after gold sputtering by SEM or imaging the water dispersed powder material at the carbon grid using TEM. In order to confirm binding of prepared nanozirconium tungestovanadate into the alginate matrix, the microscopic structure of the fabricated composite matrix was scanned using optical microscope (Olympus BX61). As an attempt to suggest the mechanism of dye sorption process onto the zirconium tungestovanadate-alginate composite matrix, the FITR spectra of free composite sheet matrix and the dye adsorbed composite sheet matrix were compared using FTIR analyzer (BRUKER, Germany).

2.5. Cation Exchange Process for Dye Decolourization. Batch experiments were performed in a set of $100 \mathrm{~mL}$ glass beakers that contains $0.15 \mathrm{~g}$ from the prepared composite matrices that contain definite amounts from the prepared nanozirconium tungestovanadate. This composite matrix was mixed at different agitated speed with $50 \mathrm{~mL}$ of fixed initial concentrations from C.I. basic violet 16 dye solutions for certain time at shaking water bath. After finishing the mixing period, the composite matrix sheet was separated using plastic forceps. The adsorbate concentrations in the initial and final aqueous solutions were measured using UV-vis spectrophotometer (7230 G, Shanghai, China) at $464 \mathrm{~nm}$. The amount of dye adsorbed on composite matrix was calculated from the difference between the initial concentration and the equilibrium one. The values of percentage removal and amount of dye adsorbed were calculated using the following relationships:

$$
\text { Dye adsorption } \%=\frac{\left(C_{0}-C_{e}\right)}{C_{0}} \times 100 \% \text {, }
$$

where $C_{0}$ is the initial dye concentration and $C_{e}$ is the final dye concentration after the treatment process.

The following formula was used to determine sorbed dye amounts per mg from the fabricated composite matrix $(q)$ either at equilibrium or at specific time $(t)$ :

$$
q_{t}=\left(C_{o}-C_{t}\right) * \frac{V}{m}
$$

where $q_{t}(\mathrm{mg} / \mathrm{g})$ is the dye adsorption capacity at time $t$, $C_{o}(\mathrm{mg} / \mathrm{L})$ is the initial dye concentration, $C_{t}(\mathrm{mg} / \mathrm{L})$ is the concentration of C.I. basic violet 16 dye in solution at time $t, V(\mathrm{~L})$ is the solution volume, and $m(\mathrm{~g})$ is the amount of immobilized cation exchange material of nanozirconium tungestovanadate.

2.6. Dye Sorption Isotherms. The dye sorption capacity of prepared zirconium tungestovanadate that immobilized onto the alginate matrix at different initial concentrations at equilibrium can be illustrated by the adsorption isotherm. Equilibrium data, commonly known as adsorption isotherms, describe how the adsorbate interacts with adsorbents and give a comprehensive understanding of the nature of interaction. It is important to optimize the design of an adsorption system. Several isotherm equations have been developed and employed for such analysis and the two important isotherms of Langmuir and Freundlich isotherms are applied through this investigation.

2.6.1. Langmuir Isotherm Model. Langmuir's isotherm may be used for monolayer adsorption onto a surface containing a finite number of identical sites and assumes uniform energies of adsorption on the surface, in addition to no transmigration of the adsorbate in the plane of the surface [12]. The Langmuir isotherm model determines the maximum capacity of the adsorbent from complete monolayer coverage of the adsorbent surface.

The Langmuir isotherm [12] is represented by the following linear equation:

$$
\frac{C_{e}}{q_{e}}=\frac{1}{q_{m} K}+\frac{C_{e}}{q_{m}},
$$

where $q_{e}$ is the solid-phase dye concentration in equilibrium with the liquid-phase concentration $C_{e}, q_{m}$ is the final dye sorption capacity (most commonly in $\mathrm{mg} / \mathrm{mg}$ ), and $K$ is an equilibrium constant (most commonly used in $\mathrm{L} / \mathrm{mg}$ ). The units of $K$ are $\mathrm{L} / \mathrm{mol}$ provided that $C_{e}$ is expressed in mole/L.

2.6.2. Freundlich Isotherm Model. Adsorbents that follow the Freundlich isotherm equation are assumed to have a heterogeneous surface consisting of sites with different adsorption potentials [11], and each type of site is assumed to adsorb molecules, as in the Langmuir equation:

$$
q_{e}=K_{f} C_{e}^{n},
$$

where $K_{f}$ is constant (function of energy of adsorption and temperature) and $n$ is a constant related to adsorption intensity.

2.7. Kinetics of Dye Cation Exchange Process. The rate at which dissolved dye is removed from the aqueous solution by solid sorbents is essential to evaluate the adsorption kinetics using theoretical models in order to design and control the sorption process units. The rate and mechanism of the dye ion exchange process onto the prepared zirconium tungestovanadate that immobilized onto the alginate matrix can be elucidated based on kinetic studies. In order to analyze the adsorption kinetics of the dye onto zirconium tungestovanadate, the pseudo-first-order and pseudo-second-order models were applied.

\section{Results and Discussion}

3.1. Nanozirconium Tungestovanadate and Alginate Composite Properties. It is well known that the efficiency of the ion exchange material toward specific pollutant is determined through its ion exchange capacity. So, the cation exchanges of the prepared nanozirconium tungestovanadate and its corresponding alginate composite matrix were accounted 
TABLE 1: Ion exchange capacity of prepared materials.

\begin{tabular}{lc}
\hline Ion exchange matrix & IEC $(\mathrm{meq} / \mathrm{g})$ \\
\hline Nanozirconium tungestovanadate & 2.92 \\
Alginate sheet matrix & 0.23 \\
$\begin{array}{l}\text { Nanozirconium } \\
\text { tungestovanadate-alginate composite } \\
\text { matrix }\end{array}$ & 3 \\
\hline
\end{tabular}

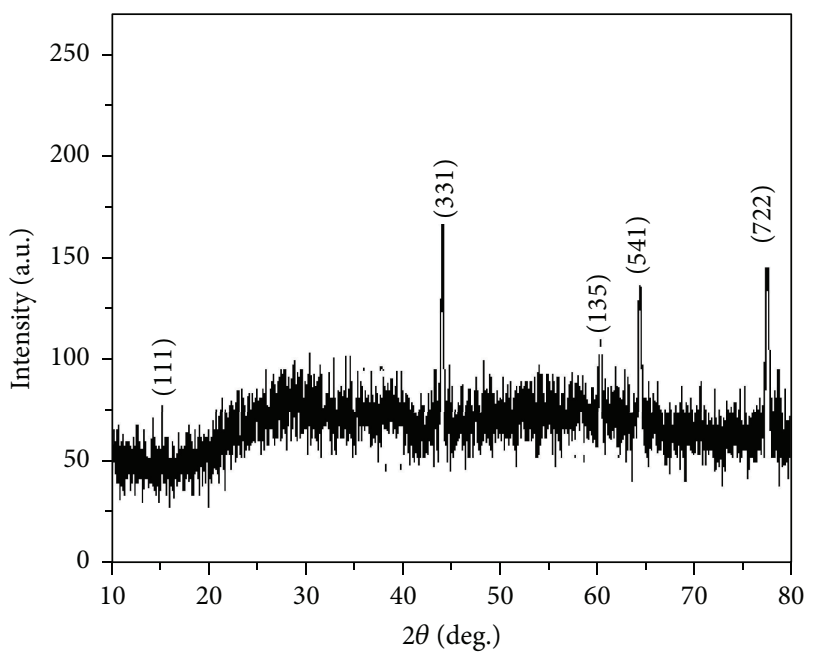

FIGURE 2: XRD patterns of fabricated zirconium tungestovanadate immobilized onto alginate matrix.

through their liberated $\mathrm{H}^{+}$that represents the main ions inside the matrices responsible for the ion exchange process. It was indicated from Table 1 that the chemically estimated cation exchange capacity of alginate matrix represents the minimum value of $0.23 \mathrm{meq} / \mathrm{g}$ compared with zirconium tungestovanadate-alginate composite matrix that records the maximum IEC of $3 \mathrm{meq} / \mathrm{g}$. Moreover, Table 1 is evident that the IEC value of prepared nanozirconium tungestovanadate material that is equal to $2.92 \mathrm{meq} / \mathrm{g}$ is extremely high compared with that of zirconium tungestovanadate produced from sol-gel technique [11]. In order determine the crystalline structure of zirconium tungestovanadate immobilized onto the alginate matrix, Figure 2 investigates the XRD pattern of the prepared composite matrices. It was indicated that it has five characteristics peaks at $2 \theta=17.4341,43.0067$, $62.351,66.0471$, and $78.8197^{\circ}$. Comparing these peaks with two reference cards of card number 01-087-1528 for zirconium tungsten oxide and card number 01-088-0586 for zirconium vanadium oxide, it was evident that the fabricated composite matrix is composed of the two materials of zirconium vanadium oxide and zirconium tungsten oxide. These results confirm that the prepared material can be identified as zirconium tungestovanadate [11]. However, small shifting in the characteristics peaks positions compared with zirconium tungsten oxide and zirconium vanadium oxide was noticed. This peak shifting may be returned to the amorphous structure of the alginate polymer matrix that immobilized the prepared zirconium tungestovanadate.

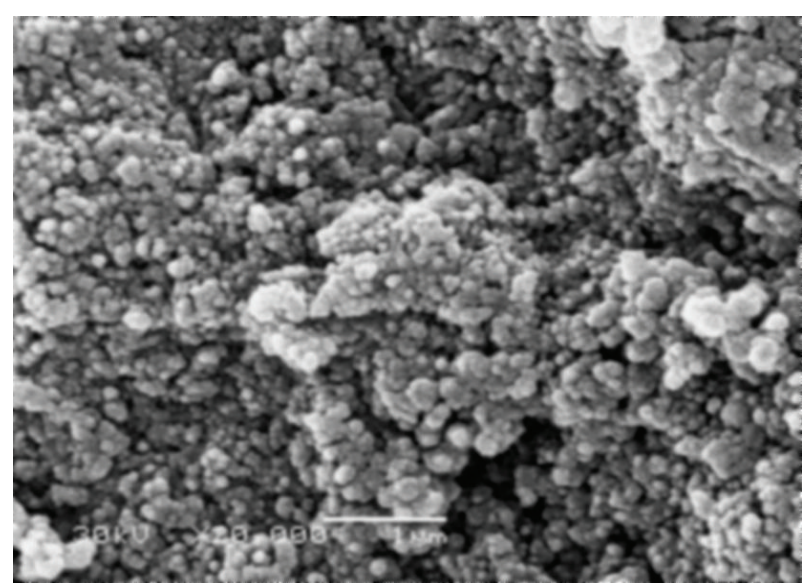

FIGURE 3: SEM image of prepared nanozirconium tungestovanadate.

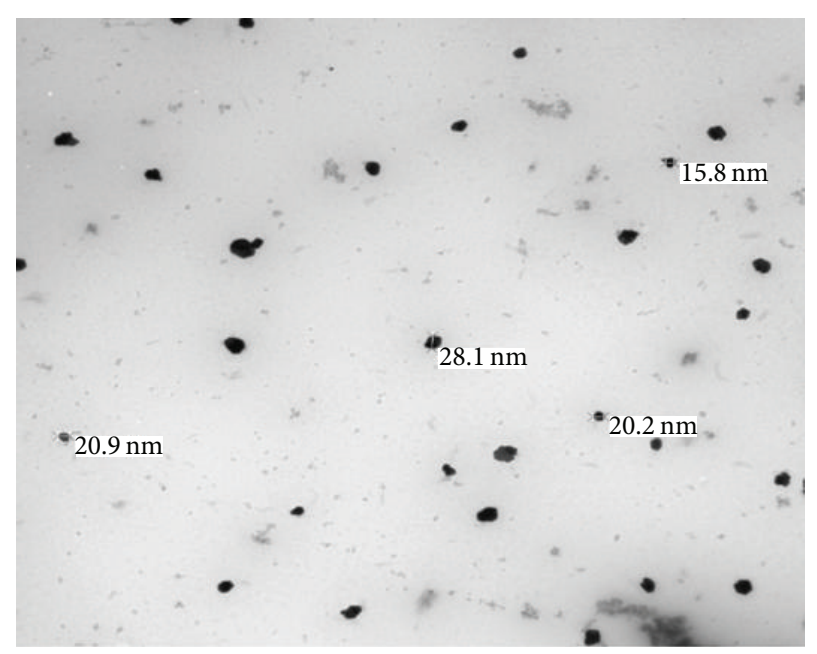

FIgURE 4: TEM image of prepared nanozirconium tungestovanadate.

With respect to the morphological structure of the prepared zirconium tungestovanadate, both SEM and TEM imaging were examined. Figure 3 illustrates the homogeneous structure of the prepared material. SEM image of zirconium tungestovanadate indicates that it was prepared in spherical morphological structure with average diameter equal to $44 \mathrm{~nm}$. However, TEM imaging (Figure 4) illustrated that the average particle diameter of the prepared cation exchange material is approximately equal to $22 \mathrm{~nm}$. The difference between the average diameters between SEM and TEM imaging may be owed to the particle aggregation that takes place during the SEM examination. Accordingly, it was evident that the prepared nanozirconium tungestovanadate produced from homogeneous precipitation technique has nanospherical morphological structure. The good nanozirconium tungestovanadate distribution onto the alginate matrix sheet was confirmed using the optical microscope. Figure 5 investigated the good distribution and dispersion of yellow powder of nanozirconium tungestovanadate onto the alginate polymer matrix. 


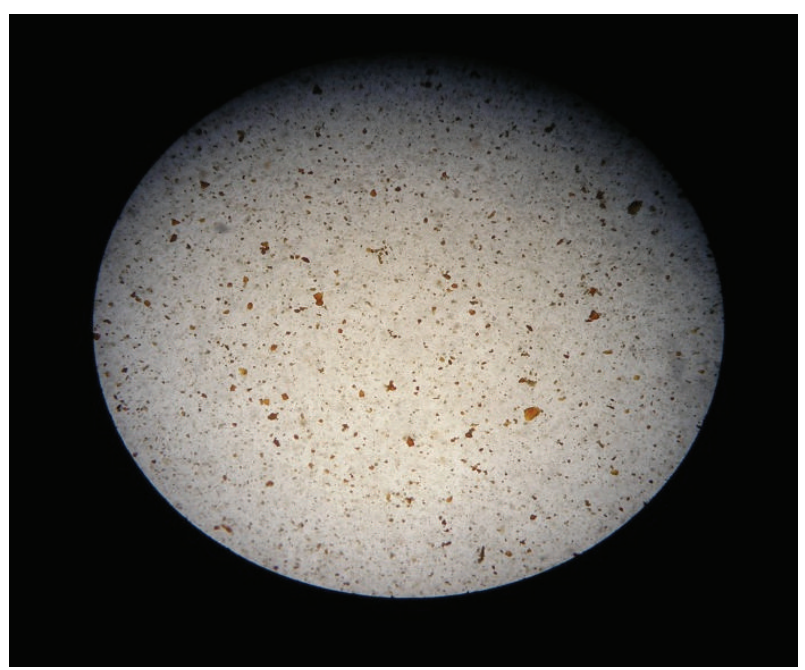

FIGURE 5: Optical microscope image of immobilized nanozirconium tungestovanadate onto alginate matrix.

3.2. Ion Exchange Process of Cationic Basic Violet 16 Decolorization Using Nanozirconium Tungestovanadate Immobilized onto Alginate Matrix. As an attempt to discuss the cation exchange behavior of the prepared nanozirconium tungestovanadate immobilized onto alginate as composite matrix at the decolorization process of the cationic basic violet 16 , the equilibrium and kinetic behaviors of the dye onto the hybrid matrix will be examined.

\subsubsection{Equilibrium Behavior of Dye Sorption Process onto} the Composite Sheet Matrix. Influence of the variation of the processing parameters on the decolourization process onto the fabricated nanozirconium tungestovanadate will be verified over determined range to elucidate the material behavior at equilibrium.

(1) Effect of Initial Dye Concentration. Figure 6(a) depicts the effect of dye concentration on the percentage sorption of basic violet 16 onto composite matrix sheet. As it is evident from the figure, the percentage sorption of dye drops with increasing initial dye concentrations. This may be attributed to the repulsive forces between the bulk phase and the dye molecules on the composite sheet surface [13]. Thus, high initial dye concentration will obstruct the movement of dye toward the free surface sites responsible for the ion exchange process.

(2) Effect of Cation Exchanger Dosage Immobilized onto Alginate Matrix. The influence of different amounts from the prepared zirconium tungestovanadate immobilized onto alginate matrix in range of $0.1-0.4 \mathrm{~g}$ against $50 \mathrm{ppm}$ dye decolorization process was tested. All experiments were carried out at the same stirring speed, $200 \mathrm{rpm}$. It was indicated from Figure 6(b) that the percentage removal of basic violet 16 was enhanced as the immobilized zirconium tungestovanadate amounts improved onto the composite matrix. The main reason for this behavior may be accounted to the greater availability of the ion exchange sites for a fixed initial dye concentration [14]. The optimum immobilized dosage from the prepared zirconium tungestovanadate to achieve $99 \%$ dye decolorization was recorded as $8 \mathrm{mg} / \mathrm{L}$.

(3) Effect of Agitation Speed. A series of batch experiments were carried out to investigate the effect of agitation speed on the basic violet 16 removal process using the fabricated zirconium tungestovanadate-alginate composite sheet. It was obvious from Figure 6(c) that the agitation speed has positive impact on the percentage dye removal. This may be accounted to the presence of a stagnant flow layer existing at the surface of the cation exchanger [15]. Increasing the stirring rate will reduce the thickness of the liquid film around the cation exchange material that declines the mass-transfer resistance to the nanozirconium tungestovanadate surface that is incorporated into the alginate matrix and enhances the cation exchange process [16].

(4) Effect of Dye Solution Acidity. Adsorption depends usually directly on electrokinetic behavior of the adsorption or ion exchange materials. Electrokinetic behavior is determined by the net charge on the particle surface of the sorbent material [13]. The effect of basic violet 16 dye solution $\mathrm{pH}$ on the cation exchange process at equilibrium onto the prepared nanozirconium tungestovanadate that was incorporated into alginate matrix was illustrated at Figure 6(d). It was indicated that the dye solution $\mathrm{pH}$ considerably affected the cation exchange process especially under acidic conditions, where the percentage dye removal onto the composite matrix was improved from $18.4 \%$ to $90.7 \%$ when the solution $\mathrm{pH}$ was increased from 1 to 12 . This result is in accordance with the expected behavior regarding the basic violet 16 which is cationic dye and has positive charge; this change may be neutralized with the available negatively charged $\mathrm{OH}^{-}$ions presence at the alkaline dye solutions $(\mathrm{pH}>7)$, in contrast to the acidic solutions that contain large amounts of positively charged $\mathrm{H}^{+}$that may compete with the cationic dye on the available exchange sites onto the prepared nanozirconium tungestovanadate immobilized onto alginate matrix. Accordingly, at the alkaline dye solutions the electrostatic interaction between the dye cation and zirconium tungestovanadate was enhanced due to the presence of excess hydroxyl groups that neutralized most of cationic dye molecules, but at acidic solutions the hydrogen ion competition results in lowering the dye sorption onto the prepared nanozirconium tungestovanadate [10] at the alginate composite matrix.

(5) Effect of Solution Temperature. The influence of the basic violet 16 dye solution temperatures onto the cation exchange process was examined at an unchanged initial dye concentration of $50 \mathrm{ppm}$. Figure 6(e) showed that the percentage dye removal increased with increasing temperatures at equilibrium. This behavior indicates that the dye sorption process onto the hybrid matrix is an endothermic process. These results may be explained as the rise at solution temperature improves the rate of diffusion of dye molecules from the solution to the zirconium tungestovanadate surface 


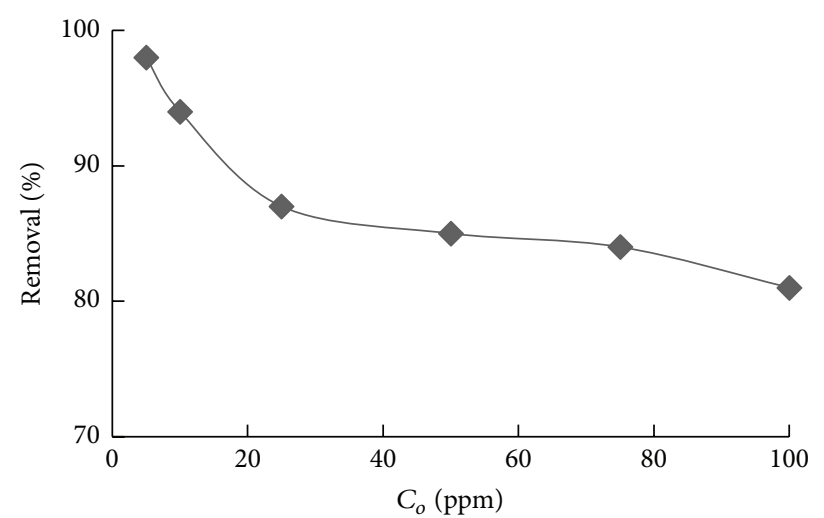

(a)

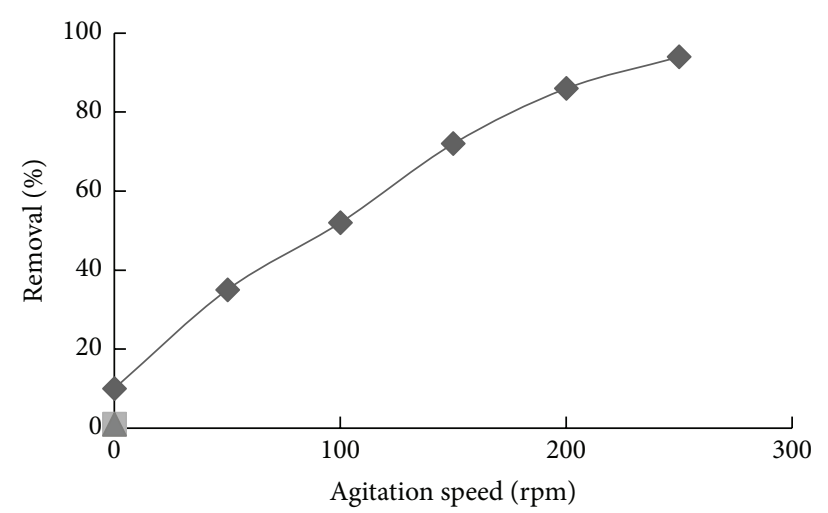

(c)

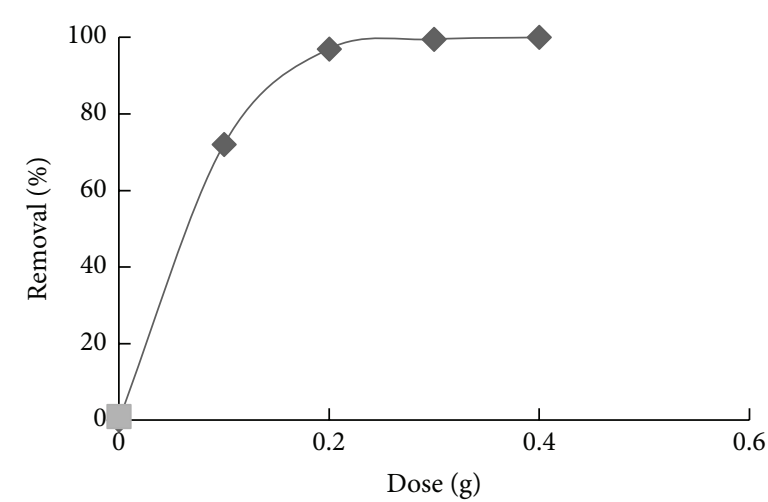

(b)

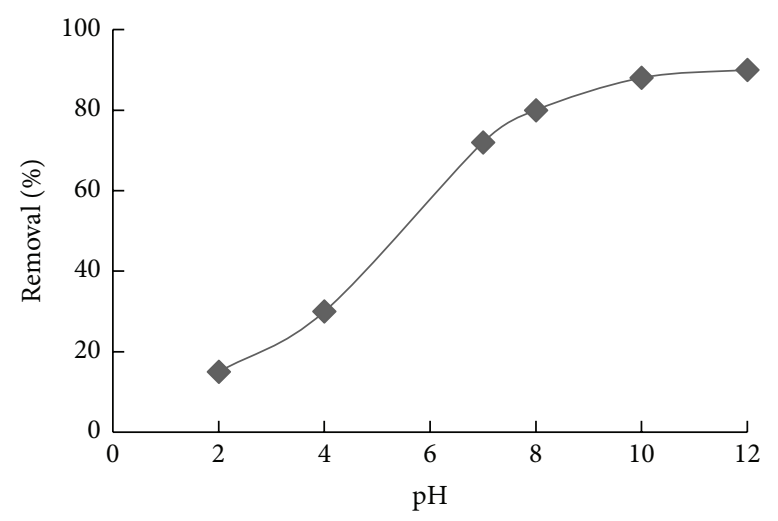

(d)

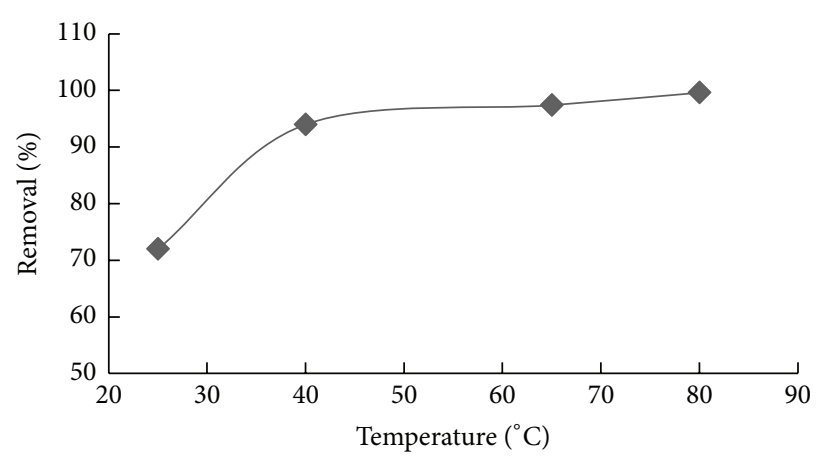

(e)

FIGURE 6: Effect of processing parameters variation on basic violet 16 decolourization process onto composite sheet matrix at equilibrium: (a) effect of initial dye concentration; (b) effect of cation exchanger dosage; (c) effect of agitation speed; (d) effect of dye solution acidity; (e) effect of solution temperature.

onto the alginate polymer matrix that enhances the cation exchange process [11].

\subsubsection{Equilibrium Modeling of Dye Sorption Process onto the Composite Sheet Matrix}

(1) Langmuir Isotherm Model. Figure 7(a) illustrates the linear plot of Langmuir equation for basic violet 16 ion exchange processes at various initial dye concentrations onto the composite sheet matrix. The Langmuir parameters for dye removal, $q_{m}$ and $K$, were calculated from the slope and intercept of this figure and tabulated in Table 2. It was evident from this table that the correlation coefficient $\left(R^{2}\right)$ value is high enough $>0.95$ to represent goodness of experimental data fitting for the linear Langmuir isotherm equation model. This result indicated that the cation exchange process of basic violet 16 dye onto composite matrix may be described well using Langmuir equation model. This result indicates that the dye removal process takes place at monolayer coverage onto nanozirconium tungestovanadate immobilized onto alginate 
TABLE 2: Estimated parameters of equilibrium isotherm models of basic violet 16 sorption onto composite sheet matrix.

\begin{tabular}{lccccc}
\hline \multirow{2}{*}{ Model parameters } & \multicolumn{2}{c}{ Equilibrium isotherm model } & \multicolumn{2}{c}{ Freundlich } \\
& $q_{m}(\mathrm{mg} / \mathrm{g})$ & $K(\mathrm{~L} / \mathrm{mg})$ & $R^{2}$ & $k_{f}$ & $n$ \\
\hline Calculated values & 10.7 & 2.3 & 0.98 & 1.8 & 0.9 \\
\hline
\end{tabular}

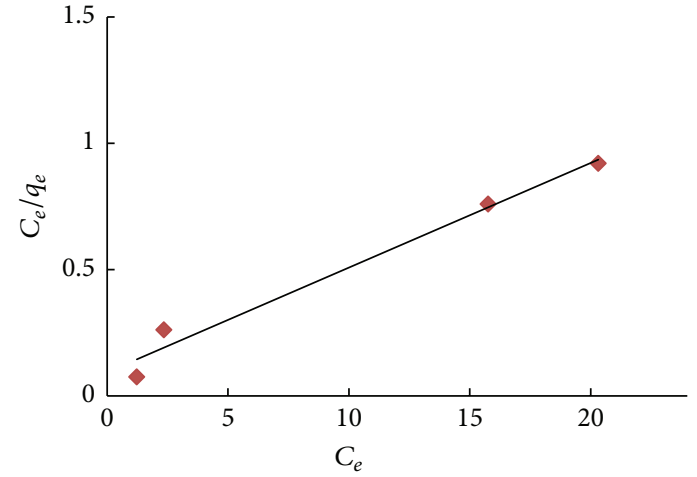

(a)

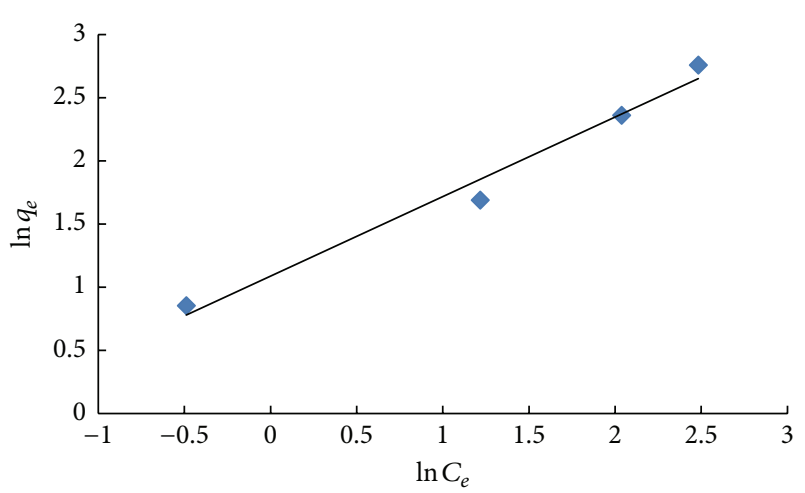

(b)

FIGURE 7: Equilibrium modeling of basic violet 16 sorption onto composite sheet matrix at various initial dye solution concentrations: (a) Langmuir; (b) Freundlich.

matrix which is homogeneous with the cation exchange process performance [17].

(2) Freundlich Isotherm Model. A straight line is obtained when plotting $\log q_{e}$ against $\log C_{e}$ which serves to evaluate the constants, $K_{f}$ and $n$. Figure $7(\mathrm{~b})$ investigates the linear regression approach for ion exchange process of basic violet 16 dye onto composite matrix to obtain the model parameters of Freundlich isotherm. From this figure according to the correlation coefficient $\left(R^{2}\right)$ value of experimental data linear fitting (Table 2), it was evident that the cation exchange process obeys the Freundlich model [14].

\subsubsection{Mechanism of Dye Decolorization Process onto Nanozir-} conium Tungestovanadate-Alginate Composite Sheet Matrix. With respect to the equilibrium modeling results, it was indicated that both Langmuir and Freundlich models fitted to the experimental data very well. These results give prediction that the uptake of the basic violet 16 onto the fabricated nanozirconium tungestovanadate-alginate hybrid matrix may take place through two parallel processes which are the ion exchange and adsorption processes. The first process is represented as the cation exchange process of the cationic dye molecules as monolayer coverage onto zirconium tungestovanadate nanoparticles incorporated into alginate matrix at the hybrid sheet, where the exchangeable $\mathrm{H}^{+}$ions inside the immobilized zirconium tungestovanadate nanoparticles were liberated into the dye solution and substituted with the positive dye molecules. However, the second process is physical adsorption process that occurs onto the surface of the hybrid matrix composed mainly of alginate polymer. According to the Freundlich assumption this physical adsorption of dye molecule onto the hybrid matrix is represented as multilayer coverage at the fabricated zirconium tungestovanadate-alginate composite. So, the dye sorption process onto the fabricated hybrid nanozirconium tungestovanadate-alginate matrix may be described as chemisorption process. As an attempt to confirm the suggested chemisorption mechanism of the dye decolorization process onto the hybrid matrix, the FTIR spectrums of the hybrid sheet matrix before and after dye sorption process were compared to detect the functionality present after the dye sorption process onto the sheet matrix. Figure 9 indicated that the FTIR spectrum of the free nanozirconium tungestovanadate-alginate matrix exhibits four characteristics transmitted bands. The corresponding zirconium tungestovanadate band was identified at the spectrum range $600-750 \mathrm{~cm}^{-1}$ that represents the superposition of metal oxygen stretching vibrations (W-O, $\mathrm{Zr}-\mathrm{O}$, and $\mathrm{V}-\mathrm{O})$ [11]. Furthermore, the deformation vibration of coordinated interstitial zirconium tungestovanadate water molecules which are responsible for the ion exchange process was recorded at $1630 \mathrm{~cm}^{-1}$ [10], whereas the band which appeared at $1419 \mathrm{~cm}^{-1}$ that corresponded to $-\mathrm{COOH}$ group is corresponding to the alginate polymer matrix [18]. Finally, the broadband at around $3500 \mathrm{~cm}^{-1}$ is typically attributed to all hydrogen bonding between $-\mathrm{OH}$ groups at the hybrid matrix that stand for either the hydroxyl groups presence at alginate matrix or $\mathrm{HOH}$ bonding of the interstitial water molecules presence inside the zirconium tungestovanadate structure. Comparing the FTIR spectrum of the free zirconium tungestovanadate-alginate hybrid matrix with that after dye sorption process (Figure 8), two new characteristics peaks were observed. These new peaks appeared at 1172 and $1373 \mathrm{~cm}^{-1}$ which is assigned for C-N stretching and C-C aromatic stretching, respectively [18]. These new peaks are 


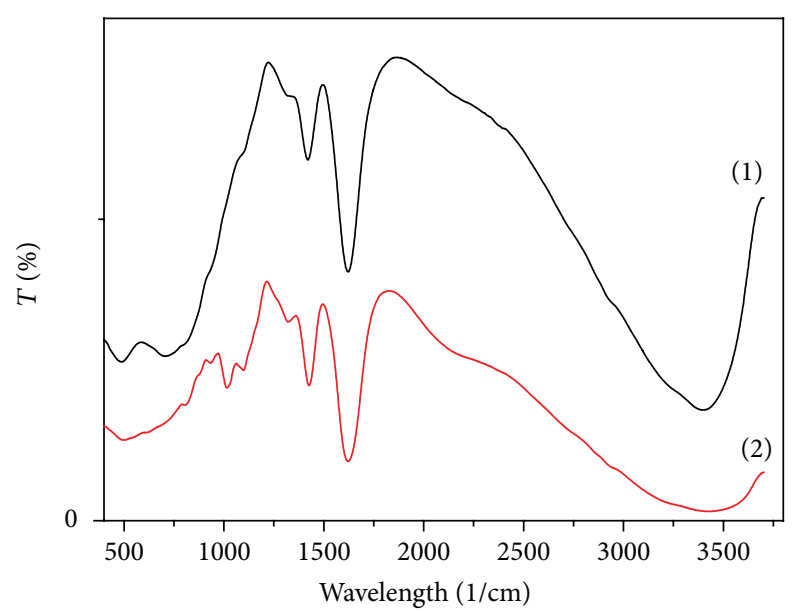

(1) Nanozirconium tungestovanadate before adsorption

(2) Nanozirconium tungestovanadate after adsoroption

FIGURE 8: FTIR spectrum of (1) free zirconium tungestovanadatealginate hybrid matrix and (2) zirconium tungestovanadate-alginate hybrid matrix after dye sorption process.

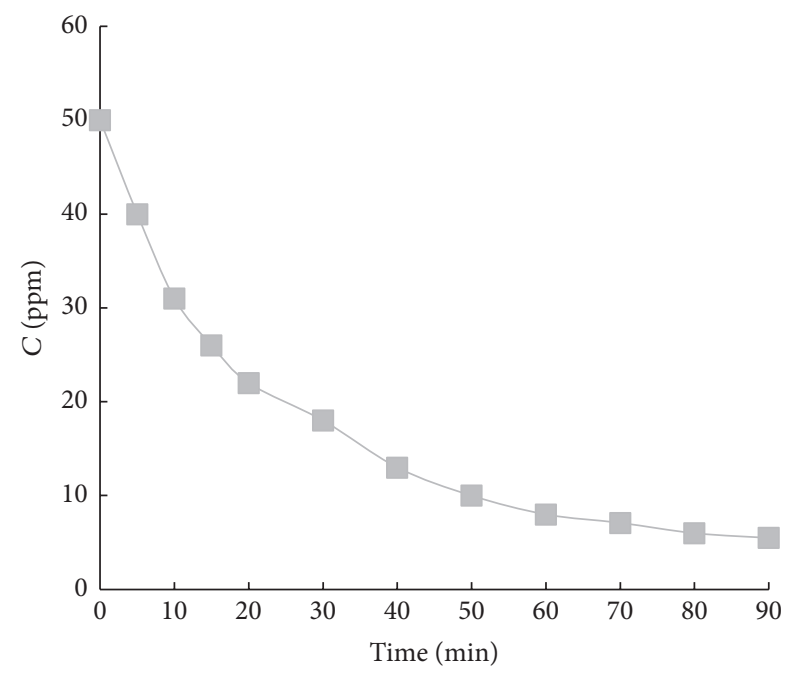

FIGURE 9: Effect of contact time on removal of basic violet 16 onto composite sheet matrix (initial dye conc. $=50 \mathrm{ppm}$; immobilized zirconium tungestovanadate dose $=0.1 \mathrm{~g}$; solution volume $=25 \mathrm{~mL}$, temperature $=25 \pm 2^{\circ} \mathrm{C} ; \mathrm{pH}=6$; agitation speed $=200 \mathrm{rpm}$ ).

characteristics for the basic violet 16 dye molecules that confirm the presence of dye on the hybrid sheet after the sorption process [19]. Moreover, comparing the FTIR spectrum of the hybrid sheet after the dye sorption process with the free one, it was indicated that the intensity degree of the broadband that appears at around $3500 \mathrm{~cm}^{-1}$ was declined after the dye sorption process. This decline gives prediction about the consumption of some hydroxyl groups at the dye sorption process. So, there is some kind of reaction that occurs between the dye molecules and the hybrid sheet matrix. This reaction may represent the ion exchange process that takes place between the $\mathrm{H}^{+}$ions presence at the interstitial water molecules inside the zirconium tungestovanadate structure and the cationic dye molecules that consumes the amount of hydroxyl groups at the hybrid sheet matrix. On the basis of the FTIR one can confirm the sorption of dye onto the hybrid sheet matrix may be described as chemisorption process.

\subsubsection{Kinetics of Dye Sorption Process onto the Composite Sheet Matrix}

(1) Effect of Contact Time. The removal of basic violet 16 dye using the fabricated composite matrix was evaluated as a function of contact time. The effect of contact time was studied through mixing $0.15 \mathrm{~g}$ of composite matrix immobilized with $0.1 \mathrm{~g}$ from the prepared nanozirconium tungestovanadate with $25 \mathrm{~mL}$ of $50 \mathrm{ppm}$ dye solution at different time intervals. Figure 9 showed that the percentage dye removal increases with contact time and attains equilibrium within 80 minutes; this effect has arisen from the fact that the amount of dye uptake is a function of time [12]. Thus, $80 \mathrm{~min}$ is selected as the reaction time required reaching equilibrium in the present dye sorption process.

(2) Kinetic Modeling of Dye Sorption Process onto the Composite Sheet Matrix. The applicability of the pseudo-first-order and pseudo-second-order models was tested for the kinetics of basic violet 16 dye sorption processes at various initial concentrations $(50,75$, and $100 \mathrm{mg} / \mathrm{L}$ ) onto the fabricated zirconium tungestovanadate-alginate composite. The kinetic rate constants were calculated using conventional rate expressions.

(2.1) Pseudo-First-Order Reaction Kinetic. Pseudo-first-order model is a simple kinetic model, which was proposed by Lagergren [14] during 1898 and is used for estimation of the surface adsorption reaction rate. The simple linear equation for pseudo-first-order reaction kinetic is given as follows:

$$
\ln \left(q_{e}-q_{t}\right)=\ln q_{e}-k_{1} t,
$$

where $k_{1}$ is the rate constant of the first-order adsorption, $q_{t}$ is the amount of dye adsorbed at time " $t$ " ( $\left.\mathrm{mg} / \mathrm{g}\right)$, and $q_{e}$ is the amount of dye adsorbed at saturation $(\mathrm{mg} / \mathrm{g})$. Plot of $\ln \left(q_{e}-q_{t}\right)$ versus $t$ allows calculation of the rate constant $k_{1}$ and $q_{e}$ for basic violet 16 dye sorption onto the fabricated composite matrix (Figure 10) [20]. The reaction rate constants, the calculated dye sorption capacities, and the linear correlation coefficients $R^{2}$ values for the different studied dye concentrations were tabulated at Table 3. Comparing the calculated dye sorption capacities with the experimental capacities values, it was indicated that the calculated values from the pseudo-first-order equation are far from their comparable experimental values. So, the pseudo-first-order reaction kinetic model is not adequate to describe the basic violet 16 sorption process onto the fabricated zirconium tungestovanadate composite matrix.

(2.2) Pseudo-Second-Order Reaction Kinetic. Pseudo-secondorder reaction kinetic can be expressed as

$$
\frac{t}{q_{t}}=\frac{1}{k_{2} q_{e}^{2}}+\frac{t}{q_{e}},
$$


TABLE 3: Parameters calculated from pseudo-first-reaction kinetic equation for basic violet 16 onto composite sheet matrix.

\begin{tabular}{lccrr}
\hline Initial dye conc. $(\mathrm{mg} / \mathrm{L})$ & $q_{e, \text { experimental }}(\mathrm{mg} / \mathrm{g})$ & $K_{1}\left(\mathrm{~min}^{-1}\right)$ & $q_{e, \text { calculated }}(\mathrm{mg} / \mathrm{g})$ & 10.8 \\
\hline 50 & 9 & 0.128 & 12.9 & 0.96 \\
75 & 12 & 0.075 & 0.95 \\
100 & 15.8 & 0.059 & 16.8 & 0.97 \\
\hline
\end{tabular}

TABLE 4: Parameters calculated from pseudo-first-reaction kinetic equation for basic violet 16 onto composite sheet matrix.

\begin{tabular}{|c|c|c|c|c|}
\hline Initial lead conc. $(\mathrm{mg} / \mathrm{L})$ & $q_{e, \text { experimental }}(\mathrm{mg} / \mathrm{g})$ & $K_{2}(\mathrm{~g} / \mathrm{mg} \cdot \mathrm{min})$ & $q_{e, \text { calculated }}(\mathrm{mg} / \mathrm{g})$ & $R^{2}$ \\
\hline 50 & 9 & 0.006 & 12.5 & 0.89 \\
\hline 75 & 12 & 0.003 & 17.5 & 0.88 \\
\hline 100 & 15.8 & $8 * 10^{-4}$ & 31 & 0.67 \\
\hline
\end{tabular}

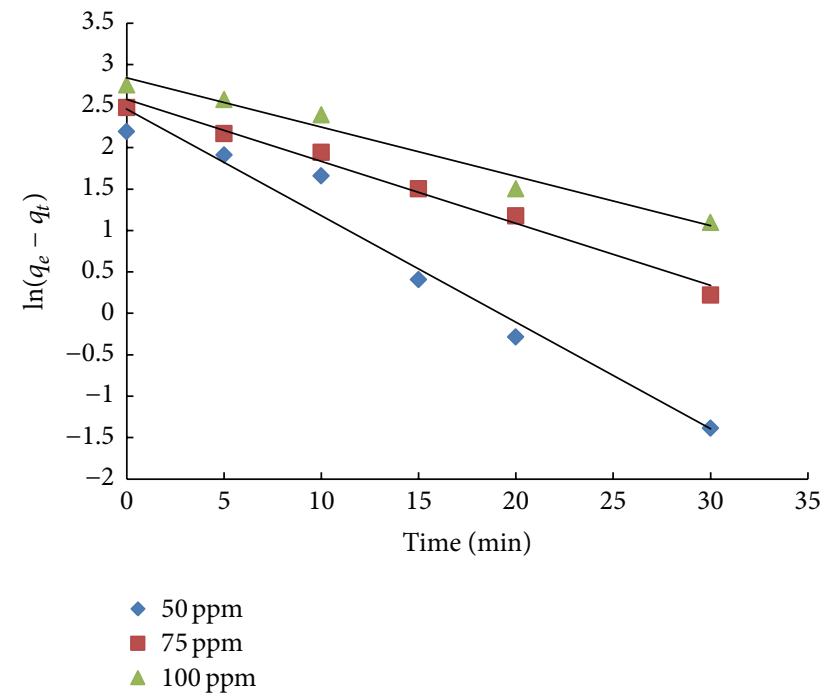

FIGURE 10: Pseudo-first-order reaction kinetics of basic violet 16 onto composite sheet matrix.

where $k_{2}(\mathrm{~g} / \mathrm{mg} \cdot \mathrm{min})$ is the pseudo-second-order rate constant, $q_{e}$ the amount adsorbed at equilibrium, and $q_{t}$ is the amount of dye adsorbed at time " $t$. " Similar to the pseudofirst-order reaction kinetic, $q_{e}$ and $k_{2}$ can be determined from the slope and intercepts of plot $t / q_{t}$ versus $t$ (Figure 11). The calculated constants from the pseudo-second-order reaction kinetic equation were illustrated at Table 4 . It was indicated that the calculated values of the dye sorption capacities at the various studied dye concentrations are adequate for the comparable experimental values. Moreover, the values of linear correlation coefficients are much higher than that of the comparable values related to the first-order kinetic model.

Accordingly, the basic violet 16 sorption process onto the prepared zirconium tungestovanadate-alginate composite matrix is following the pseudo-second-order reaction kinetic model. This result confirms that the decolourization process involves chemisorptions mechanism [8].

\section{Conclusion}

The present investigation deals with the efficiency of the homogeneous precipitated nanozirconium tungestovanadate

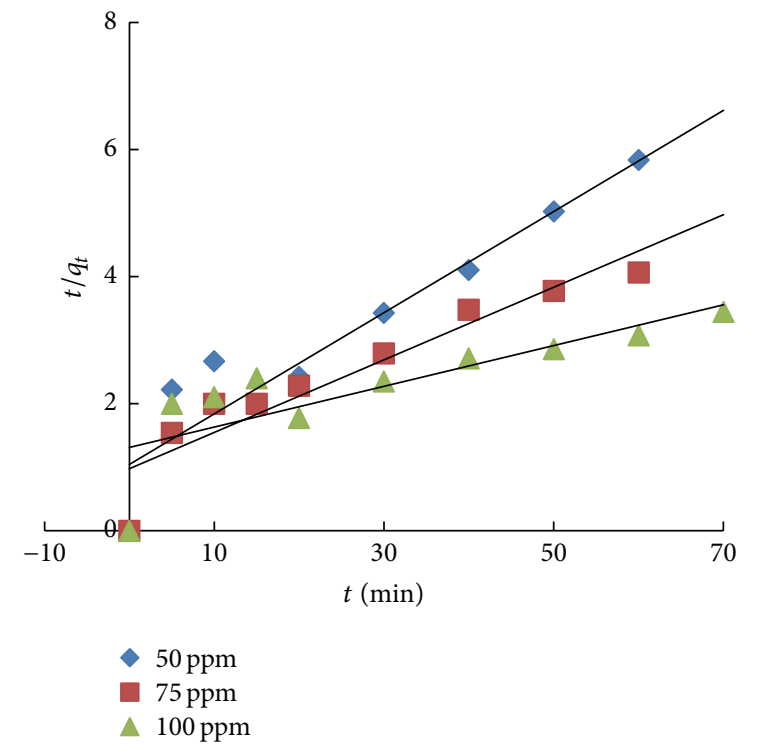

FIGURE 11: Pseudo-second-order reaction kinetics of basic violet 16 onto composite sheet matrix.

immobilized onto alginate matrix as a novel cation exchanger for basic violet 16 decolorization. The decolorization behavior onto the composite matrix revealed that as the contact time increased, the dose of the intended adsorbate of the adsorption efficiency increased, as well. The equilibrium dye sorption data fitted very well in the Langmuir and Freundlich isotherms equations. These results indicated that the decolorization process takes place as monolayer and multilayer coverage onto the fabricated composite matrix. The FTIR analysis of the composite matrix before dye sorption process suggested that the dye decolorization is chemisorption process. The kinetic modeling of basic violet 16 onto the composite matrix sheet was described by pseudo-secondorder equations confirming the chemisorptions nature of the decolorization process. This study concludes that the zirconium tungestovanadate-alginate composite sheet could be employed as an appropriate, accessible, and efficient cation exchange material for the removal of different cationic dye pollutants from aquatic environments. 


\section{Conflict of Interests}

The authors declare that there is no conflict of interests regarding the publication of this paper.

\section{Acknowledgment}

This work was supported by the Egyptian Science and Technology Development Fund (STDF) (Grant no. 5546).

\section{References}

[1] S. Chatterjee, M. W. Lee, and S. H. Wooa, "Adsorption of congo red by chitosan hydrogel beads impregnated with carbon nanotubes," Bioresource Technology, vol. 101, no. 6, pp. 1800$1806,2010$.

[2] H. Shokry Hassan, A. B. Kashyout, I. Morsi, A. A. A. Nasser, and A. Raafat, "Fabrication and characterization of gas sensor micro-arrays," Sensing and Bio-Sensing Research, vol. 1, pp. 3440, 2014.

[3] E. Chatzisymeon, N. P. Xekoukoulotakis, A. Coz, N. Kalogerakis, and D. Mantzavinos, "Electrochemical treatment of textile dyes and dyehouse effluents," Journal of Hazardous Materials, vol. 137, no. 2, pp. 998-1007, 2006.

[4] G. Ciardelli, L. Corsi, and M. Marcucci, "Membrane separation for wastewater reuse in the textile industry," Resources, Conservation and Recycling, vol. 31, no. 2, pp. 189-197, 2001.

[5] I. K. Konstantinou and T. A. Albanis, " $\mathrm{TiO}_{2}$-assisted photocatalytic degradation of azo dyes in aqueous solution: kinetic and mechanistic investigations, a review," Applied Catalysis B: Environmental, vol. 49, no. 1, pp. 1-14, 2004.

[6] K. P. Gopinath, S. Murugesan, J. Abraham, and K. Muthukumar, "Bacillus sp. mutant for improved biodegradation of Congo red: random mutagenesis approach," Bioresource Technology, vol. 100, no. 24, pp. 6295-6300, 2009.

[7] E. Forgacs, T. Cserháti, and G. Oros, "Removal of synthetic dyes from wastewaters: a review," Environment International, vol. 30, no. 7, pp. 953-971, 2004.

[8] V. K. Garg, R. Gupta, A. B. Yadav, and R. Kumar, "Dye removal from aqueous solution by adsorption on treated sawdust," Bioresource Technology, vol. 89, no. 2, pp. 121-124, 2003.

[9] H.-Y. Zhu, R. Jiang, L. Xiao, and W. Li, "A novel magnetically separable $\gamma-\mathrm{Fe}_{2} \mathrm{O}_{3} /$ crosslinked chitosan adsorbent: preparation, characterization and adsorption application for removal of hazardous azo dye," Journal of Hazardous Materials, vol. 179, no. 1-3, pp. 251-257, 2010.

[10] M. M. Abd El-Latif and M. F. Elkady, "Synthesis, characterization and evaluation of nano-zirconium vanadate ion exchanger by using three different preparation techniques," Materials Research Bulletin, vol. 46, no. 1, pp. 105-118, 2011.

[11] M. F. Elkady, E. M. El-Sayed, H. A. Farag, and A. A. Zaatout, "Assessment of novel synthetized nanozirconium tungstovanadate as cation exchanger for lead ion decontamination," Journal of Nanomaterials, vol. 2014, Article ID 149312, 11 pages, 2014.

[12] H. Shokry Hassan, M. F. Elkady, A. H. El-Shazly, and H. S. Bamufleh, "Formulation of synthesized zinc oxide nanopowder into hybrid beads for dye separation," Journal of Nanomaterials, vol. 2014, Article ID 967492, 14 pages, 2014.

[13] G. Crini and P.-M. Badot, "Application of chitosan, a natural aminopolysaccharide, for dye removal from aqueous solutions by adsorption processes using batch studies: a review of recent literature," Progress in Polymer Science, vol. 33, no. 4, pp. 399447, 2008.

[14] Z. Aksu, A. I. Tatli, and Ö. Tunç, "A comparative adsorption/biosorption study of Acid Blue 161: effect of temperature on equilibrium and kinetic parameters," Chemical Engineering Journal, vol. 142, no. 1, pp. 23-39, 2008.

[15] M. Wawrzkiewicz, "Removal of C.I. Basic Blue 3 dye by sorption onto cation exchange resin, functionalized and nonfunctionalized polymeric sorbents from aqueous solutions and wastewaters," Chemical Engineering Journal, vol. 217, pp. 414425, 2013.

[16] L. Wang, J. Zhang, and A. Wang, "Removal of methylene blue from aqueous solution using chitosan-g-poly(acrylic acid)/montmorillonite superadsorbent nanocomposite," Colloids and Surfaces A: Physicochemical and Engineering Aspects, vol. 322, no. 1-3, pp. 47-53, 2008.

[17] L. Zhou, J. Jin, Z. Liu, X. Liang, and C. Shang, "Adsorption of acid dyes from aqueous solutions by the ethylenediaminemodified magnetic chitosan nanoparticles," Journal of Hazardous Materials, vol. 185, no. 2-3, pp. 1045-1052, 2011.

[18] A. S. Sartape, A. M. Mandhare, V. V. Jadhav, P. D. Raut, M. A. Anuse, and S. S. Kolekar, "Removal of malachite green dye from aqueous solution with adsorption technique using Limonia acidissima (wood apple) shell as low cost adsorbent," Arabian Journal of Chemistry, 2013.

[19] A. M. Aljeboree, A. N. Alshirifi, and A. F. Alkaim, "Kinetics and equilibrium study for the adsorption of textile dyes on coconut shell activated carbon," Arabian Journal of Chemistry, 2014.

[20] S. Lagergren, "About the theory of so-called adsorption of soluble substances," Kungliga Svenska Vetenskapsakademiens Handlingar, vol. 24, pp. 1-39, 1898. 

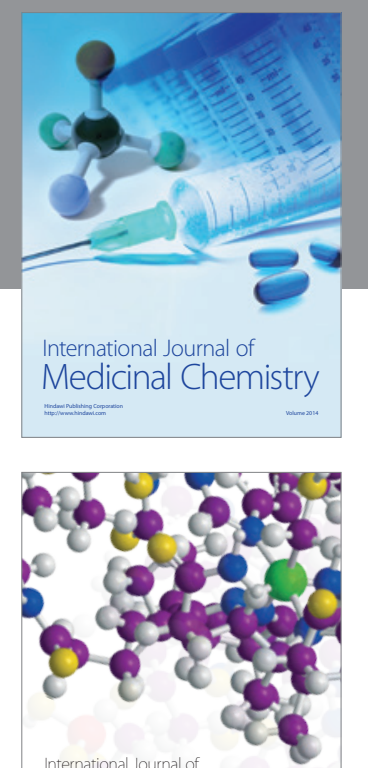

\section{Carbohydrate} Chemistry

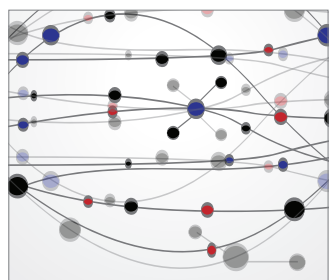

The Scientific World Journal
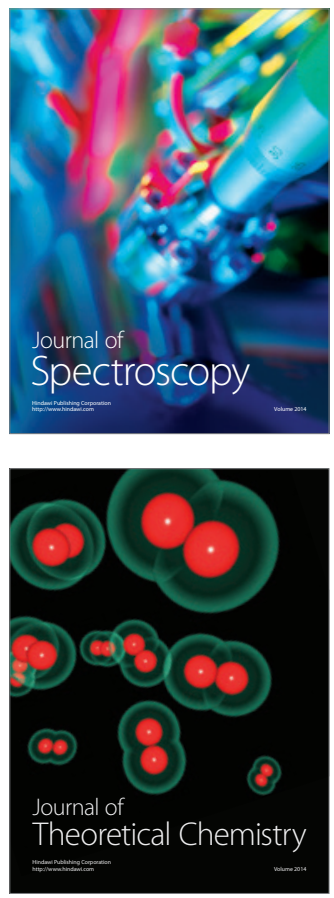
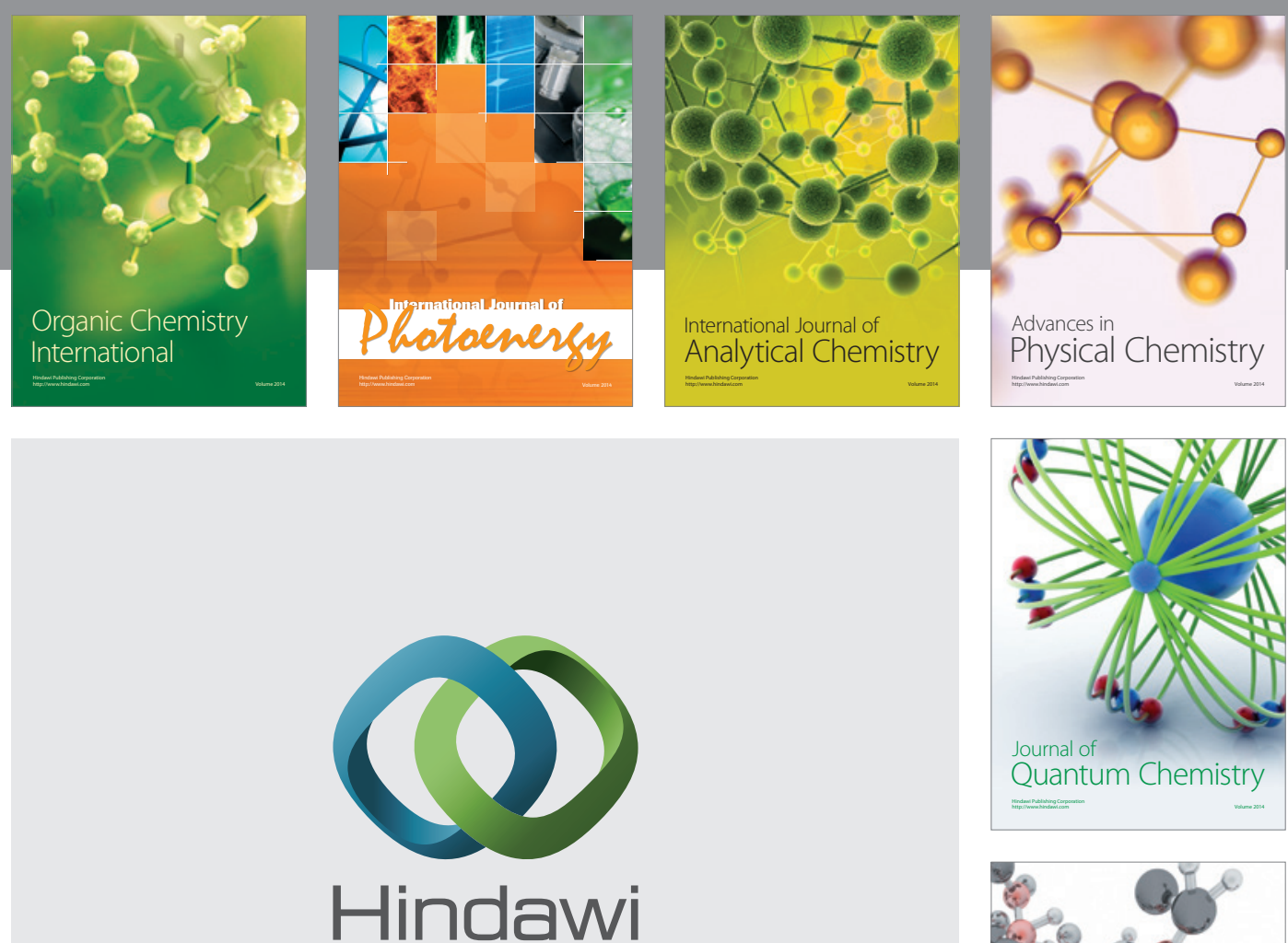

Submit your manuscripts at

http://www.hindawi.com

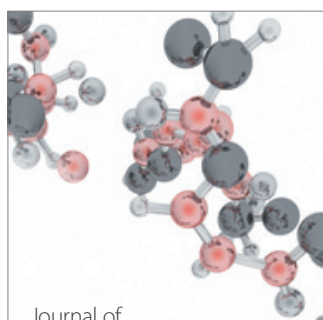

Analytical Methods

in Chemistry

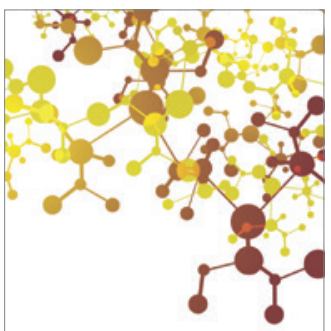

Journal of

Applied Chemistry

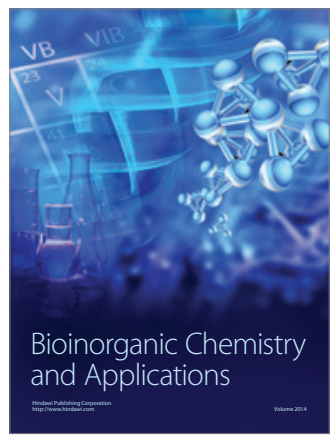

Inorganic Chemistry
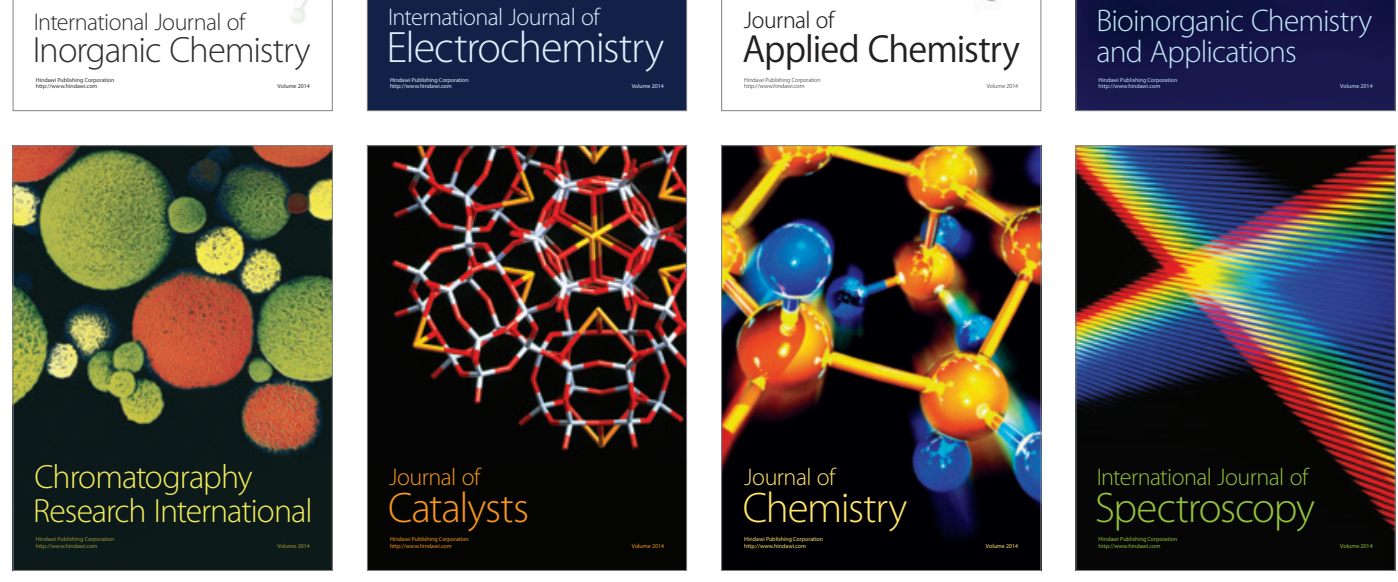